\title{
Accesibilidad usando las tecnologías de la información y la comunicación
}

\author{
Yorka Ortiz Ruiz \\ Magister en Informática Educativa \\ Académico Línea Informática Educativa \\ Universidad de Los Lagos, Chile \\ Email:yortiz@ulagos.cl
}

Recepción: 27-09-2016 / Aceptación: 24-11-2016

\section{Resumen}

La sociedad del conocimiento o de la información está en constante evolución, y afecta la mayor parte de la población mundial en todos los ámbitos de la vida cotidiana. Esto trae aparejado el surgimiento de diferentes dispositivos tecnológicos cada vez más sofisticados, pero que rápidamente quedan obsoletos por el surgimiento de otros más atractivos en términos de diseño o aplicaciones novedosas.

Uno de los aspectos fundamentales de la sociedad del conocimiento es tener acceso a gran cantidad de información disponible en los diferentes medios, teniendo la posibilidad de compartirla con un número ilimitado de personas por medio de las redes virtuales, lo que puede generar grandes ventajas. Es así como el objetivo de este trabajo fue analizar los elementos que facilitan y dificultan la accesibilidad de la información, no sólo de personas con discapacidad o necesidad educativa especial, sino a todo público o usuario que necesita acceder a información contenida en un documento, una presentación o un sitio web; aspectos que no siempre son conocidos, considerados o valorados por quienes tienen la gran responsabilidad de transmitir información.

Palabras clave: accesibilidad, educación, tecnologías, sociedad de la información.

\begin{abstract}
Knowledge or information society is evolving constantly, and it affects the greatest part of the world's population in all the areas of daily life. This brings harnessed the appearance of different technological devices which are every time more sophisticated but which quickly become obsolete because of the appearance of others that are more attractive in terms of their design or innovative apps.

One of the essential aspects of the knowledge society is to have access to a great amount of available information in the different media, having the chance to share it with an unlimited number of people through virtual networks, which can generate big advantages. This is how the objective of this work was to analyze the elements that facilitate and hinder the accessibility to the information not only to disable people or people in needs of special education but also to all people or users who need access to information contained in documents, a presentation or a web site; that is, to access aspects that are not always known, considered or valued from the people who have the great responsibility of transmitting information.
\end{abstract}

Key Words: accessibility, education, technologies, knowledge society. 


\section{Introducción}

La sociedad actual, Sociedad de la Información o del Conocimiento, según Hargreaves (2003), se caracteriza por la constante y abundante circulación de información, a la cual debieran tener acceso todos los ciudadanos que la requieren, considerando que el nivel o calidad de la misma, dependerá de la capacidad del propio ciudadano en discriminar qué es lo más importante.

Lo expuesto anteriormente plantea que la incorporación de las tecnologías de información y comunicación (TIC) en los distintos ámbitos de la vida cotidiana, supone una ventaja tanto por la cantidad como por la variedad de información y experiencias que de otra forma estarían fuera del alcance de muchas personas; por lo mismo, al presentar una condición de discapacidad, los avances tecnológicos significarían una ayuda adicional para superar las barreras de participación en todo ámbito.

Para muchas personas desenvolverse en la cotidianidad significa un reto en forma permanente, más aún para quienes tienen una condición de discapacidad, ya sea física o sicológica, producto del avance en edad o por una condición de nacimiento. Los avances tecnológicos no significan disminuir las barreras de accesibilidad, las cuales pueden deberse a inadecuados diseños, falta de sensibilidad hacia estas necesidades o simplemente desconocer condiciones básicas y dispositivos, que permiten aumentar el público objetivo que pueda acceder a dicha información.
En una sociedad cambiante en la que la información se expande de manera rápida $\mathrm{y}$ circula continuamente, se puede asegurar que la educación se somete directamente a esta realidad. Hargreaves (2003) menciona que las escuelas no son inmunes a este contexto, $y$ en un mundo en constante cambio, con un conocimiento en expansión y comunidades en transformación, los docentes deben, por lo tanto, desarrollar capacidades para correr riesgos y manejar cambios cuando se les plantean de forma repetida nuevas demandas $y$ nuevos problemas.

Ante esta realidad surgen varias interrogantes ¿Los estudiantes acceden a la información que se quiere transmitir usando recursos tecnológicos?, ¿se tiene en consideración que, eventualmente, se puede tener un estudiante con alguna necesidad educativa especial en el aula? En definitiva, ¿se conocen los elementos que hacen accesible la información que se quiere transmitir, se está preparado para estos desafíos producto de la inclusión? Son preguntas que casi nunca se hacen; sin embargo, para una gran cantidad de personas resulta muy difícil o imposible usar la tecnología, recurriendo a apoyos técnicos o personas que cumplan la función de intermediarios. 


\section{Metodología}

\section{Definición y concepto de accesibilidad de la información}

$\mathrm{Al}$ analizar el estado actual en torno a la accesibilidad de la información a través de las TIC, hay que partir por clarificar el concepto. La Real Academia Española (2016) define la cualidad de accesible como "que tiene acceso", “de fácil acceso o trato" o "de fácil comprensión, inteligible”. Según el observatorio de la accesibilidad (s.f.), esta se puede definir como la cualidad de fácil acceso para que cualquier persona - incluso aquellas que tengan limitaciones en la movilidad, en la comunicación o el entendimiento - pueda llegar a un lugar, objeto o servicio. Esta concepción concuerda con la planteada por Fundación ONCE (s.f.), que señala que la accesibilidad es la condición que deben cumplir los entornos, procesos, bienes, productos y servicios, así como los objetos o instrumentos, herramientas y dispositivos para ser comprensibles, utilizables y practicables por todas las personas en condiciones de seguridad y comodidad; y de la forma más autónoma y natural posible. Presupone la estrategia de diseño para todos y se entiende, sin perjuicio de los ajustes razonables, que deban adoptarse.

En su Manual de accesibilidad de la Corporación Ciudad Accesible de Boudeguer y Squella ARQ (2010), se señala que accesibilidad es el conjunto de características que debe disponer un entorno urbano, edificación, producto, servicio o medio de comunicación para ser utilizado en condiciones de comodidad, seguridad, igualdad y autonomía por todas las personas; incluso por aquellas con capacidades motrices o sensoriales diferentes. Una buena accesibilidad es aquella que pasa desapercibida a los usuarios.

Esta accesibilidad desapercibida implica algo más que ofrecer una alternativa al peldaño de acceso: busca un diseño equivalente para todos, cómodo, estético y seguro. Es sinónimo de calidad y seguridad, siendo este último requisito fundamental en el diseño. Si carece de seguridad en el uso para un determinado grupo de personas, deja de ser accesible. La gran ventaja de la accesibilidad desapercibi$d a$ es el valor agregado que otorga al diseño, ya que no restringe su uso a un tipo o grupo etario de personas. Los entornos, productos o servicios pueden ser usados con comodidad por todos a lo largo de la vida.

En Chile, a nivel ministerial, se establece el concepto de accesibilidad universal (Ministerio de Planificación, 2011) dentro de la Ley 20.422, como la condición que deben cumplir los entornos, procesos, bienes, productos y servicios, así como los objetos e instrumentos, herramientas y dispositivos, para ser comprensibles, utilizables y practicables por todas las personas, en condiciones de seguridad y comodidad, de la forma más autónoma y natural posible.

\section{Accesibilidad versus usabilidad}

La accesibilidad no sólo significa o implica la necesidad de facilitar el acceso a la información, sino también a la de facilitar el uso 
(usabilidad) del servicio, producto, objeto, herramienta, iniciativa, dispositivo, etc.

Es importante distinguir la diferencia entre estos dos conceptos: usabilidad y accesibilidad. El primero, centrado en la perspectiva del usuario, hace referencia a la facilidad de uso e interacción entre estos y las tecnologías; el segundo, lo que persigue es que cualquier usuario, con independencia de sus condiciones personales o materiales, pueda aprender todos los contenidos de esta tecnología. Cuando se habla de accesibilidad y usabilidad, nos referimos a tecnologías que pueden ser de utilidad, tanto para personas con discapacidad o con necesidades educativa especiales, como para usuarios que no presentan dichas condiciones.

Para el Servicio Nacional de la Discapacidad (2016), la accesibilidad representa "Un desafío para el Chile actual, las barreras físicas, sociales y tecnológicas debieran constituir un aspecto a considerar en la planificación de programas y acciones gubernamentales; y por cierto, del mundo privado". Según la Organización Internacional para la Estandarización (ISO, 2016) y el Centro de Información y Documentación Científica (2010), usabilidad es "la capacidad de un software de ser comprendido, aprendido, usado y ser atractivo para el usuario en condiciones específicas de uso”.

A partir de esta conceptualización (Pantoja, 2004), ISO plantea ciertos principios básicos que sustentan la usabilidad de una herramienta, aplicación o producto: a. Facilidad de aprendizaje: focalizada en que nuevos usuarios puedan tener una interacción efectiva. Está relacionada con la predictibilidad, sintetización, familiaridad, generalización de los conocimientos previos y la consistencia.

b. Facilidad de Uso: se concentra en que el usuario hace uso de la herramienta, con menos pasos y más naturales a su formación específica. Tiene que ver con la eficacia y eficiencia de la herramienta.

c. Flexibilidad: variedad de posibilidades con las que el usuario y el sistema pueden intercambiar información. Además, implica la posibilidad de diálogo, la multiplicidad de vías para realizar la tarea, similitud con tareas anteriores y la optimización entre el usuario y el sistema.

d. Robustez: nivel de apoyo al usuario que facilita el cumplimiento de sus objetivos. Está relacionada con la capacidad de observación del usuario, recuperación de información y de ajuste de la tarea al usuario.

De acuerdo con (Bevan y Mcleod, 1994), estos principios se aplican y/o adaptan a tecnologías en general, software y hardware, colaborando en varias tareas y características que favorecen el aprendizaje, disminuyen los costos de asistencia y ayuda al usuario; optimizan costos de diseño, rediseño y mantenimiento de recursos; aumenta la tasa de conversión de visitantes, mejora la imagen y el prestigio del recurso; mejora la calidad de vida de los usuarios, ya que reduce su estrés e incrementa la satisfacción y la productividad. 


\section{Las TIC y oportunidad de inclusión}

Las tecnologías de la información y la comunicación son consideradas herramientas al alcance de un gran número de personas, disminuyendo así las barreras de accesibilidad, en especial de personas con algún grado de discapacidad, permitiendo una mejor calidad de vida y mejorando su personalidad al sentirse más integrados y participantes activos de la sociedad.

En el ámbito de las TIC, la accesibilidad apunta al conjunto de propiedades que debe incorporar un producto, servicio o sistema, de forma que el mayor número posible de personas, y en el mayor número posible de circunstancias, puedan acceder a él y usarlo. Como lo señala De Larra (2007), las tecnologías de la información y la comunicación "han supuesto barreras para la integración y la normalización de personas con discapacidad", siendo que este grupo en particular es el que puede verse más beneficiado con el uso y aplicación de las TIC. Al respecto De la Larra (2007), menciona que se debe considerar que si bien éstas plantean un medio revolucionario y alternativo de interacción con el mundo, también podrían representar un "obstáculo sino se aplican los principios básicos de Accesibilidad y/o Diseño para Todos”.

En torno a la responsabilidad en el acceso a la tecnología ésta no sólo recae en los desarrolladores, diseñadores o programadores web, sino en todo usuario habitual de estas, la cual se hace especialmente relevante en quienes están ligados al ámbito educativo, sea este a nivel básico, medio o superior; considerando la diversidad del público objetivo que atienden los docentes, quienes no solo tienen relación con estudiantes ligados a Proyectos de Integración; es decir, que presentan algún grado de discapacidad.

$\mathrm{Al}$ analizar un recurso tecnológico, considerando características de accesibilidad, no solo se debe remitir a personas con discapacidad reconocida, ya sea física o sicológica, sino también personas adultas, con problemas de vista o de oído; problemas visuales asociados a determinados colores como el daltonismo (Academia Americana de Oftalmología, 2016), y otros problemas visuales desconocidos para la mayoría de las personas que no están ligadas a temas de salud.

Es una realidad que a medida que las personas envejecen, cambia la forma en que los sentidos (gusto, olfato, tacto, vista y oído) pueden entregar información del medio. Esto significa que los sentidos se vuelven menos agudos y pueden presentar problemas para diferenciar los detalles. Estos cambios pueden llevar a cambiar el estilo de vida, afectando su calidad a medida que avanzan los años, presentando problemas para comunicarse, para disfrutar las diferentes actividades e involucrarse con las personas de su entorno.

Todos los sentidos pueden resultar afectados por la edad, pero el efecto mayor es en la audición y la visión. Muchos de estos cambios se pueden compensar con aparatos como anteojos y audífonos o por cambios en el estilo de vida (MedlinePlus, 2016). Por todo lo 
anterior, se puede considerar que el acceso a la información es una necesidad en todos los escenarios, tanto en el ámbito familiar, educativo, laboral y social, lo que obliga a estar preparados tanto a los receptores y más aún a los responsables de entregar información al público objetivo. Lo anterior en el caso específico de quienes ejercen docencia en cualquier nivel, porque queda claro que en todas las edades se enfrentan dificultades en el acceso a la información.

Toledo (2006), enumera algunas de las razones que justifican la introducción de la tecnología de ayuda en las aulas ordinarias y de apoyo:

a. Los nuevos avances tecnológicos proporcionan nuevas opciones a los alumnos con necesidades educativas especiales para participar y realizar tareas de enseñanza y aprendizaje.

b. Permite que los alumnos alcancen sus potencialidades. Los alumnos con necesidades especiales no solo tienen discapacidades, es necesario potenciar sus habilidades $\mathrm{y}$ aprovecharlas.

c. Ayudan a muchos alumnos a acceder a la información, interactuar con otros y participar en actividades desarrolladas en la Red a las que no podrían acceder sin el uso de esta tecnología.

d. Las alternativas digitales del e-learning presentan oportunidades al alumno con necesidades educativas especiales para explorar y beneficiarse de estos recursos a través del uso de la tecnología asistida. e. El uso de la tecnología les motiva, aumenta su autoestima, hace que no se sientan diferentes a los demás compañeros.

f. Los ordenadores ofrecen retroalimentación al alumno sobre sus errores, pero no les hacen comentarios negativos ni críticas que les puedan desmotivar.

g. Para Beltrán (2004), la tecnología aplicada a las personas con discapacidad sólo es efectiva cuando:

h. Parte de evaluaciones que determinan los programas que necesitan.

i. Resulta adecuada a su potencial: necesidades, fuerzas y debilidades.

j. La dificultad de la tarea se ajusta a sus capacidades y experiencias.

k. Tiene en cuenta su experiencia lingüística.

1. Suministra feedback inmediato sobre el progreso del alumno.

Al realizar un análisis en torno a las condiciones de accesibilidad de los recursos tecnológicos que debemos preparar para garantizar una difusión adecuada, consideraremos las condiciones o lineamientos que se establecen desde ámbitos ministeriales o instituciones ligados al tema, para así dar cuenta del escenario de apoyo o reconocimiento de esta característica o condición de acceso universal.

Se partirá por responder la siguiente pregunta: ¿qué papel desempeña el gobierno con respecto a los retos y demandas que exigen 
el acceso a la información? En Chile, desde la década de los años 90, al interior de los colegios se han venido desarrollando los denominados Proyectos de Integración Escolar, que pretenden dar respuestas educativas a niños con necesidades educativas especiales dentro de una educación regular (Decreto № 1/98 y No 1300/02), trayendo como consecuencia, que cada vez sean más amplias las ofertas educativas para atender esta diversidad. Esta experiencia cuenta con el respaldo de normativas que la avalan.

Uno de estos decretos es el conocido D.U. 170/2009, su implementación regula lo dispuesto en la Ley 20.201/2007 (Ministerio de Educación, 2016), respecto de la evaluación diagnóstica integral de aquellos estudiantes que presentan necesidades educativas especiales $(\mathrm{NEE})^{1}$ de carácter transitoria, que reúnan las condiciones para participar en un Programa de Integración Escolar y que accedan a la subvención de necesidades educativas especiales de carácter transitorio; y respecto de la evaluación diagnóstica integral de los estudiantes con NEE de carácter permanente que accedan al incremento de la subvención especial diferencial.

Estos antecedentes avalan una política que busca la integración de estudiantes con necesidades educativas especiales, lo que se traduce en un nuevo reto para el docente de educación básica y media, que va más allá de entregar sus conocimientos habituales, sino que también va hacia atender, en conjunto con un educador diferencial, a estudiantes que participan de estos proyectos de integración.

\section{Apoyo a la integración de las TIC en la aten- ción a la diversidad}

El Ministerio de Educación chileno (s.f.) cuenta con espacios en línea que permiten el acceso a materiales tecnológicos (recursos educativos digitales) para atender a la diversidad de estudiantes, los cuales se acompañan con las respectivas fichas de trabajo, con el objetivo de contribuir al desarrollo de aprendizajes, a través de la integración de las Tecnologías de la Información y la Comunicación (TIC) en los establecimientos educacionales de Educación Especial o con Programas de Integración. En total son 38 recursos a disposición de educadores, pero sobre esta propuesta de recursos educativos, no existe evidencias de evaluación sobre su calidad, variedad y uso pedagógico efectivo, sino sólo una ficha de apoyo didáctico, sin tener en cuenta aspectos básicos como seguimiento de aplicación, cuenta de descargas, fecha de actualización, entre otros.

Junto a lo anterior, en el marco del día internacional de la sordera, la Subsecretaria de Educación, Valentina Quiroga (Ministerio de Educación, 2014), hizo anuncios importantes para los niños con necesidades educativas especiales; además de la ampliación de un piloto

1. A lo largo del artículo, la autora también se referirá a este término mediante sus siglas. 
de uso de TIC para apoyar proceso formativo de niños con discapacidades auditivas, y la creación de un piloto de TIC para el proceso educativo de niños con NEE múltiples.

Por otra parte, la Pontificia Universidad Católica de Chile (PUC) cuenta con un Centro de Desarrollo de Tecnologías de Inclusión (CEDETi, 2016). El sitio web de este centro ofrece una gama de recursos descargables junto a guías de uso, información sobre sus avances, opciones de capacitación, entre otros temas. El trabajo de CEDETi se enfoca en: desarrollar software educativos de primer nivel, que sean libres, gratuitos y accesibles para el usuario final; mantener un sistema de comunicación e información permanente con la comunidad; dictar programas de formación continua para profesionales que trabajan en áreas vinculadas con educación y discapacidad; ofrecer apoyo, acompañamiento y asesoría para desarrollo de estrategias educativas inclusivas.

Al realizar la pregunta: ¿cuáles iniciativas o propuestas se conocen que estén encaminadas a promover la creación de documentos accesibles para mitigar o eliminar las barreras que existen actualmente?

Existe una Convención sobre los Derechos de las Personas con Discapacidad de la Organización de las Naciones Unidas, a la que Chile se suscribió en el año 2008. En esta convención los Estados se comprometen, entre otros aspectos, a proporcionar información que sea accesible para las personas con discapacidad y asegurar el acceso de éstas, en igualdad de condiciones, a la información y las comunicaciones.

De esta forma, el Servicio Nacional de la Discapacidad, SENADIS, ${ }^{2}$ promueve y fomenta el desarrollo de documentos, contenidos y tecnologías accesibles con el fin de que las personas con discapacidad puedan vivir en forma independiente, y participar plenamente en todos los aspectos de la vida. Para esto estableció una GUÍA DE DOCUMENTOS ACCESIBLES, fácilmente descargable desde la web y que entrega en forma muy clara y precisa las herramientas y recomendaciones que se deben realizar en distintos archivos para hacerlos accesibles a cualquier usuario.

Todo se agrupa en lo que se denomina accesibilidad universal, que SENADIS (s.f.) define como condición que deben cumplir los entornos, procesos, bienes, productos y servicios, así como los objetos o instrumentos, herramientas y dispositivos, para ser comprensibles, utilizables y practicables por todas las personas, en condiciones de seguridad y comodidad, de la forma más autónoma y natural posible.

El mismo SENADIS establece lineamientos para la accesibilidad en diferentes aspectos o modalidades de acción, los cuales clasifica en entornos, tecnologías inclusivas y accesibilidad web (Ministerio de Educación, s.f.). De esta forma se puede establecer que existen en-

2. La autora también utilizará las siglas de esta institución para referirse a ella. 
tidades que se preocupan de colaborar en este proceso de accesibilidad universal, no tan solo para estudiantes con necesidades educativas especiales, sino también para personas que presentan algún tipo de condición que dificulta el acceso a la información.

\section{Diseño para todos o diseño universal}

Es una constante que los diseñadores web, de productos, servicios y otros conciban sus creaciones para un individuo estándar, que cumpla con los parámetros antropométricos medios. Así puede ser adecuado para una persona de edad media, peso medio, altura media, capacidad intelectual media, contextura media, etc., pero la realidad es que estos ideales no son efectivos para la masificación de un producto o servicio, sin pensar en la realidad cultural, social y biológica de determinadas latitudes; y sobre todo atender a toda la diversidad. Esto puede deberse a intereses económicos que buscan la masificación y la rentabilidad.

El objetivo del diseño para todos o diseño universal es su intento de maximizar al número de potenciales usuarios que puedan interactuar con éxito con un entorno, producto o servicio virtual. Esto en definitiva es acercar lo más posible este recurso, producto, servicio, objeto, instrumento, dispositivo o herramienta al individuo fuera de la media descrita en el párrafo anterior, lo cual hace más difícil adecuar un diseño a las capacidades funcionales, y aquí es cuando se recurre a las adaptaciones y/o ayudas técnicas.
A partir de esto se pueden plantear ciertos principios en torno al diseño, esto lo define De la Larra (2007) como diseño para todos. El Center for Universal Design (2016), (Universidad de Carolina del Norte) plantea siete principios, los cuales son:

a. Uso equitativo: diseño útil y accequible para personas con diversas capacidades.

b. Uso flexible: adaptable a un amplio rango de preferencias y capacidades individuales.

c. Simple e intuitivo: fácil de entender, independiente de la experiencia, conocimiento, nivel cultural o capacidad de concentración.

d. Información perceptible: transmite de forma eficaz la información necesaria al usuario, con independencia de las condiciones ambientales y de su capacidad sensorial.

e. Tolerancia a los errores: minimiza el peligro y las consecuencias negativas producidas por acciones accidentales e involuntarias.

f. Bajo esfuerzo físico: debe poder ser usado de forma cómoda y eficiente con el mínimo esfuerzo.

\section{g. Espacio suficiente de aproximación y} uso: las dimensiones y el espacio deben ser apropiados para permitir el acercamiento, alcance, manipulación y uso, independientemente del tamaño del cuerpo, postura o movilidad del usuario. 


\section{Brecha digital y Accesibilidad}

En la sociedad actual denominada del conocimiento o de la información, ya sea para una persona, empresa u organización, el poder acceder a las tecnologías de la información y comunicaciones (TIC) es un requisito importante para participar de una sociedad cada vez más dependiente de estas herramientas.

El término brecha digital se refiere a la distancia entre quienes pueden hacer uso efectivo de las herramientas de información y comunicación y los que no pueden, por ser personas mayores, con discapacidad, analfabetos y/o analfabetos tecnológicos; o personas con limitaciones económicas o en situación marginal (Gutiérrez, 2001). Herskovic (2016), en su ensayo Software libre y Brecha Digital, señala que el concepto de brecha digital se usa para cuantificar la diferencia existente entre sectores que tienen acceso a las herramientas de la información y aquellos que no lo tienen.

La brecha digital puede analizarse en distintos contextos, por ejemplo, el económico, social, educacional, etc., y entre diferentes grupos como por ejemplo, entre países, sectores y personas. Una clasificación posible de la brecha digital es brecha digital internacional, que compara la brecha digital entre países; brecha digital interna (doméstica), que estudia la brecha digital al interior de las naciones, por ejemplo, entre estratos socioeconómicos, según tamaño de empresas o geografía.

En un contexto escolar, abordar la brecha digital pasa por conseguir la máxima utiliza- ción de los recursos informáticos, tanto para atender al alumnado con necesidades educativa específicas, como para la normalización de las TIC de uso común (diseño para todos); y la preparación / formación del profesorado en su transformación, uso y aprovechamiento. Esto contempla la adquisición y adaptación de hardware y software adecuado a las necesidades de este alumnado; garantizando la disponibilidad de tecnologías de ayuda a la comunicación aumentativa para los alumnos que lo precisen; $y$ fomentando el diseño accesible en la elaboración de recursos (tanto comunes como específicos) multimedia y servicios de red e internet. Todo esto impulsa la formación y la creación de grupos de trabajo, seminarios y proyectos de innovación e investigación educativa cuyas líneas de acción se centren en la utilización y/o el análisis, catalogación y evaluación de las TIC en la atención a la diversidad (Soto y Fernández, 2003).

\section{Desafíos para una accesibilidad universal}

La accesibilidad universal implica la inclusión de todas las personas aunque tengan algún tipo de discapacidad, considerando que el foco no está en hacer contenidos paralelos o diferenciados de acuerdo al tipo de estudiante, sino de hacer contenidos accesibles desde un principio, asumiendo los principios del diseño para todos.

Si nos planteamos desafíos en torno a barreras de accesibilidad hay que tener en cuenta el Libro Blanco de $\mathrm{I}+\mathrm{D}+\mathrm{i}$ al servicio de las personas con discapacidad y las personas mayores, propone dos estrategias complementa- 
rias: El diseño para todos o diseño universal y el desarrollo de ayudas técnicas. La primera de las estrategias, consiste en diseñar productos y servicios que puedan ser utilizados por el mayor número posible de personas, sin necesidad de llevar a cabo una adaptación o diseño especializados. La segunda de ellas se aplica cuando, a pesar de intentar implementar la anterior, sigue siendo necesario desarrollar productos y servicios concretos o ayudas técnicas, específicamente destinadas a las personas con discapacidad, con el objeto de compensar sus limitaciones funcionales. De esta forma, esta estrategia se aplica cuando la reducción de la habilidad o capacidad para manejar un producto alcanza un nivel tal, que impide su utilización.

Otros aspectos a considerar como desafíos tienen que ver con precisar una política eficaz y práctica sobre las tecnologías de la información y la comunicación aplicada a la educación, en la cual se aborden en forma precisa la educación diferencial y el diseño de recursos educativos atendiendo a la diversidad. Este desafío por tanto apunta a la formación de los futuros pedagogos como una política transversal, considerando el escenario con el cual se encontrarán en términos regulares, diversidad de estudiantes, como con los proyectos de integración escolar, los cuales afectan a todas las especialidades, tanto de enseñanza pre básica, básica y media.

Considerando la relevancia de los elementos analizados esto debería ser una política establecida desde el Ministerio de Educación para la formación de profesionales de todas las áreas o disciplinas, porque la transmisión eficiente de la información no sólo es una capacidad que deben desarrollar los educadores sino todo profesional y de todas las instituciones formadoras, ya sean públicas como privadas.

\section{Conclusiones}

Los avances tecnológicos que caracterizan a la actual sociedad supondrían mejorar la comunicación y el acceso a la información de la mayor parte de la comunidad, pero esto no siempre es la realidad. Las TIC deberían ser herramientas al servicio de las personas, en especial para aquellas que presenten algún tipo de discapacidad; sin embargo, no son considerados por diseñadores de productos y servicios, menos por quienes desean transmitir información relevante para su formación o conocimiento. No se consideran términos como accesibilidad desapercibida, accesibilidad universal y usabilidad, siendo aspectos claves de relevar en el desarrollo de materiales o espacios dispuestos para la transmisión de información. Autores como Toledo (2006) y Beltrán (2004) ya exponían razones sólidas para integrar las TIC en educación, y específicamente aplicadas a discapacidad.

Existen lineamientos planteados desde entidades ministeriales y otras organizaciones preocupadas del tema, siendo el servicio nacional de la discapacidad (SENADIS) una de las entidades mandatadas a liderar estos aspectos, y que plantean la accesibilidad como una prioridad, para esto proponen mejoras 
en el diseño y así hacerlo accesible para todo tipo de persona. Esto se conoce como diseño para todos o diseño universal, el cual contribuye a mejorar las condiciones de uso y la calidad de vida de los usuarios al maximizar al número de potenciales usuarios que pueden interactuar con éxito con el entorno, producto o servicio. Esto se ratifica por el hecho de que Chile es uno de los países adscritos, desde el año 2008, a la convención sobre derechos de las personas con discapacidad de la ONU.

Las tecnologías contribuyen a la integración social y laboral de las personas con discapacidad. Existe una creciente concientización de la sociedad, la cual parte en el aula por medio de los programas de integración escolar (PIE), que buscan incluir en un ambiente regular a un estudiante con alguna necesidad educativa especial. Para esto, en Chile existen normativas ministeriales específicas como el Decreto 170 del año 2009 que regula la implementación de lo dispuesto en la Ley 20.201 del año 2007, lo que viene a plantear un desafío a los docentes de especialidad que deben trabajar a la par con los educadores diferenciales, ya que deben interactuar en forma permanente con estudiantes con necesidades educativas especiales en una aula regular.

En definitiva, la masificación de las tecnologías requiere de diversas estrategias cohesionadas para garantizar su accesibilidad, medidas legales que garanticen la no discriminación, medidas sociales que sensibilicen a la ciudadanía, educativas para habilitar a las personas con discapacidad a utilizar las TIC con normalidad. Esto permitiría aprovechar los beneficios, ventajas y nuevas oportunidades que nos ofrecen estas tecnologías en la sociedad de la información, disminuyendo así la denominada brecha digital, que es la distancia en el acceso a la información en distintos escenarios.

Plantear lo anterior en la formación de futuros docentes y profesionales de otras áreas debiese ser una prioridad de las instituciones formadoras, considerando la realidad actual que busca disminuir la discriminación e impulsar la inclusión como un aspecto cultural asumido por naciones que luchan por justicia e igualdad.

\section{Referencias}

Academia Americana de Oftalmología (2016). ¿Qué es el daltonismo? Recuperado de: http:// www.geteyesmart.org/eyesmart/diseases-es/daltonismo/

Bevan, N. y Mcleod, M. (1994). Medición de la Usabilidad en Contexto, Comportamiento y Tecnología de la Información, Reino Unido. Recuperado de: http://www.nigelbevan.com/papers/music94.pdf 
Chile, Ministerio de Educación (s.f.) Medidas de Accesibilidad e Inclusión para personas con discapacidad. Recuperado de http://www.senadis.gob.cl/pag/138/435/documentos_y_herramientas

Chile, Ministerio de Educación. (s.f.) Recursos educativos digitales. Recuperado de http:// www.educacionespecial.mineduc.cl/index3.php?id_portal=20\&id_seccion=4813\&id_contenido $=25803$

Chile, Ministerio de Educación. (2014). Recuperado de http://www.mineduc.cl/contenido_int. php?id_contenido=29617\&id_portal=1\&id_seccion $=10$

Chile, Ministerio de Educación (2016). Normativa Educación Especial. Recuperado de: http://www.educacionespecial.mineduc.cl/index2.php?id_portal=20\&id_seccion=3084\&id_ contenido $=12269$

Chile, Ministerio de Desarrollo Social (s.f.). Líneas de acción. SENADIS. Recuperado de: http:// www.senadis.gob.cl/pag/132/591/descripcion_general

Chile, Ministerio de Planificación (2016). Ley 20422. Establece normas sobre igualdad de oportunidades e inclusión social de personas con discapacidad. Recuperado de: http://www.leychile.cl/Navegar?idLey=20422

Chile, Ministerio de Trabajo y Asuntos Sociales. (2003) Libro Blanco I + D + I al servicio de las Personas con Discapacidad y las Personas Mayores.

Chile, Servicio Nacional de la Discapacidad e Instituto Nacional de Estadísticas. (2004) Primer Estudio Nacional de la Discapacidad en Chile, Conclusiones. Capítulo 4. (En línea). Recuperado de: http://www.ine.cl/canales/chile_estadistico/encuestas_discapacidad/pdf/conclusiones4.pdf

Confederación Española de Personas con Discapacidad Física y Orgánica (Cocemfe) (s.f.). Observatorio de la accesibilidad Recuperado de: http://www.observatoriodelaaccesibilidad.es/accesibilidad/accesibilidad/definicion/

Corporación Ciudad Accesible. Boudeguer y Squella ARQ (2010).Manual de Accesibilidad Universal. Recuperado de: http://www.ciudadaccesible.cl/wp-content/uploads/2012/06/manual_accesibilidad_universal1.pdf

De la Larra, M. (2007). Cuaderno 7: Discapacidad y Accesibilidad. Fundación Orange. España. Recuperado de http: //fundacionorange.es/documentos/análisis/cuadernos/cuaderno_4_abri.pdf.

España, Centro de Información y Documentación Científica (2010). Normas de calidad para las revistas electrónicas propuestas por el CINDOC en Revistas Científicas Electrónicas. Recuperado de http://www.erevistas.csic.es/especial_revistas/revistas74.htm 
España, Normas de calidad para las revistas electrónicas propuestas por el CINDOC en Revistas Científicas Electrónicas. (2010). Recuperado de: http://www.erevistas.csic.es/especial_revistas/revistas74.htm

Fundación Once (s.f.). Guía de responsabilidad social empresarial y discapacidad de la Fundación ONCE. Recuperado de: http://rsed.fundaciononce.es/glosario.html

Gutiérrez, E. (2001). La educación en Internet e Internet en la educación como factor de la brecha digital. Congreso la Educación en Internet e Internet en la Educación. Ministerio de Educación. Madrid. Recuperado de: http://www.inclusiondigital.net/ponen/brecha/Overview.html

Hargreaves, A. (2003). Enseñar en la sociedad del conocimiento, España: Barcelona. Editorial Octaedro.

Herskovic, V. (s.f.). Software Libre y Brecha Digital. Facultad de Ciencias Físicas y Matemáticas. Universidad de Chile. Recuperado de: http://users.dcc.uchile.cl/ cfuenzal/recursos/brecha_digital_sol.pdf

MedlinePlus (2016). Cambios en los sentidos con la edad. Recuperado de http://www.nlm.nih. gov/medlineplus/spanish/ency/article/004013.htm

Observatorio de la Accesibilidad (s.f). Recuperado de http://www.observatoriodelaaccesibilidad.es/accesibilidad/accesibilidad/definicion/

Pantoja, J. C. (2004). Arquitectura de Información: Introducción al proceso de Desarrollo en el Diseño de Interfaces de Usuario (Draft). Recuperado de: http://www2.udec.cl/ai/proceso.pdf

Pontificia Universidad Católica de Chile (s.f.). Centro UC Tecnologías de Inclusión CEDETI. Recuperado de: http://www.cedeti.cl/

Real Academia Española (2016). Diccionario de la Lengua Española Recuperado de: http:// lema.rae.es/drae/?val=

Soto, F.J. y Fernández, J (2003) Realidades y Retos de la Inclusión Digital. Comunicación y Pedagogía.

Toledo. P. (2006). El profesor en el proceso de selección de tecnología de apoyo para alumnos con NEE. Comunicación y pedagogía: Nuevas tecnologías y recursos didácticos. 